\title{
ISOLAMENTO DE FUNGOS EM ESMALTES UTILIZADOS EM SALÃO DE BELEZA
}

\author{
ISOLATION OF FUNGI IN NAIL POLISHES USED IN BEAUTY \\ SALON
}

\author{
Pujol $^{1}$, J. R. S. C., Livramento ${ }^{2}$, A., Botelho' ${ }^{2}$ T. K. R. \\ Afiliações: 1- Curso de Biomedicina, Fundação Universidade Regional de Blumenau (FURB), Blumenau, Santa Catarina, Brasil. \\ 2 - Departamento de Ciências Farmacêuticas, Fundação Universidade Regional de Blumenau (FURB), Blumenau, Santa Catarina, Brasil. \\ Endereço do autor de correspondência Rua São Paulo, Itoupava Seca, Blumenau, Santa Catarina, Brasil. CEP 89030-000. \\ tatianibotelho@furb.br
}

\section{Resumo}

A manipulação de materiais biológicos por profissionais como manicures e pedicures implica no risco de transmissão de diversos microrganismos. Neste sentido, este trabalho teve como objetivo analisar a contaminação fúngica em esmaltes provenientes de salões de beleza. Foram analisadas 101 amostras de esmaltes de diferentes cores e marcas comerciais de quatro salões de beleza. Os esmaltes foram cultivados em meio contendo Agar Sabouraud dextrosado, com e sem inibidores. A identificação presuntiva das leveduras foi feita através de triagem em meio cromogênico. Assim, observou-se o crescimento fúngico em 2,97\% dos esmaltes analisados. $O$ gênero Candida foi o único fungo isolado das amostras. Através do método cromogênico, identificou-se duas cepas como Candida krusei e uma como Candida spp. Os resultados deste estudo demostraram que os esmaltes podem ser um fômite para a transmissão de agentes fúngicos.

Palavras-chave: Fungos; onicomicoses; esmaltes; salões de beleza.

\section{Abstract}

The manipulation of biological materials by professionals such as manicures and pedicures implies the risk of transmission of various microorganisms. In this sense, the objective of this work was to analyze the fungal contamination in nail polishes from beauty salons. It were analyzed 101 samples of nail polishes of different colors and trademarks of four beauty salons. The nail polishes were grown in culture medium containing dextosed Sabouraud Agar, with and without inhibitors. Presumptive identification of yeasts was done by screening in chromogenic medium. Thus, fungal growth was observed in $\mathbf{2 . 9 7 \%}$ of the nail polishes analyzed. The genus Candida was the only fungus isolated from the samples. Through the chromogenic method, two strains were identified as Candida krusei and one as Candida spp. The results of this study demonstrated that the nail polishes may be a fomite for the transmission of fungal agents.

Keywords: Fungi; onychomycosis; nail polishes; beauty salons. 
Introdução

Prevalentes em todo o mundo, as micoses superficiais fúngicas são comuns em países tropicais como o Brasil. Nestas regiões, as altas temperaturas e a umidade facilitam a proliferação destes agentes, o que culmina, inclusive, em problemas sanitários ${ }^{1}$. Os fungos podem aderir à camada superficial da pele, mucosas, regiões cutaneomucosas e nas unhas, causando as infecções ungueais ou onicomicoses ${ }^{2}$.

No que tange às onicomicoses é importante ressaltar que suas principais causas são infecções por espécies de Candida spp. e fungos dermatófitos do gênero Trichophyton,

\section{Epidermophyton e Microsporum ${ }^{3}$. A} transmissão destes referidos microrganismos ocorre de forma direta e indireta. A primeira delas diz respeito ao contato pessoa a pessoa; a segunda forma ocorre por meio de fômites ${ }^{4}$.

As espécies do gênero Candida pertencem à microbiota humana, e são encontrados em diversos sítios anatômicos. São oportunistas, geralmente patogênicos em pacientes imunodeprimidos. No entanto, a infecção por este fungo é comum, apresentando uma frequência de $80 \%$. As lesões por Candida variam de cutâneas a sistêmicas ${ }^{5,6}$.
Profissionais como manicures e

pedicures, cotidianamente, manipulam materiais biológicos para a remoção do eponíquio em salões de beleza. Deste modo, o compartilhamento de utensílios e materiais perfurocortantes aumenta o risco de contágio de bactérias, vírus e fungos. Todavia, sabe-se que muitos desses profissionais não estão suficientemente capacitados para o exercício de tal profissão ou desconhecem as medidas de biossegurança ${ }^{7,8}$.

Neste sentido, o presente trabalho teve como objetivo analisar a contaminação fúngica em esmaltes utilizados em salões de beleza.

\section{Métodos}

Trata-se de uma pesquisa do tipo experimental que buscou analisar o crescimento fúngico a partir de amostras de esmaltes provenientes de salões de beleza no período de julho a novembro de 2017. Durante o processo em questão, a análise contou com 101 amostras de esmaltes de diferentes cores e marcas comerciais de quatro salões de beleza da região do Vale do Itajaí, estado de Santa Catarina, as quais foram analisadas no Laboratório Micologia da Fundação Universidade Regional de Blumenau - FURB. 
Para dar prosseguimento à pesquisa, os esmaltes de uso diário nos salões de beleza foram separados por cor, marca e quantidade do produto. As amostras foram classificadas de acordo com o volume de produto contido em cada um dos frascos: pouco, médio e cheio.

Para o cultivo, empregaram-se os meios de cultura ágar Sabouraud dextrosado com e sem inibidores. Ademais, as amostras foram semeadas em placa de petri, com o próprio pincel do esmalte e incubadas na estufa a temperatura de $25^{\circ} \mathrm{C}$ num período de 15 a 30 dias. Todas as culturas foram observadas diariamente ao longo de 30 dias. A amostra positiva, além da diferenciação macroscópica das colônias, foi analisada microscopicamente.

O processo de identificação presuntiva das leveduras foi feito através de triagem em meio HiCrome TM Candida diferencial ágar, um meio cromogênico capaz de identificar e diferenciar algumas espécies pela coloração das colônias. Além do mais, foi realizado o controle positivo e negativo com esmaltes sem uso pessoal. O controle positivo foi feito por contaminação de um esmalte, com uma cepa de Candida albicans e semeado em Agar saboraud dextrosado com visualização de crescimento fúngico após 15 dias de incubação em estufa a $25^{\circ} \mathrm{C}$. O controle negativo, por sua vez, foi realizado por semeadura de esmalte em Agar saboraud dextrosado sem visualização de crescimento fúngico após 15 a 30 dias de incubação na estufa a $25^{\circ} \mathrm{C}$.

\section{Resultados}

Dentre os esmaltes analisados, três apresentaram positividade para crescimento fúngico (2,97\%). Além disso, os esmaltes a partir dos quais se notou crescimento fúngico foram de tons mais escuros, sendo dois da cor rosa e um laranja. As amostras positivas, importante realçar, não foram das mesmas marcas comerciais. No entanto, todas eram provenientes do mesmo salão de beleza. Um dos esmaltes que apresentaram positividade para a contaminação fúngica encontrava-se fora do prazo de validade.

Os resultados deste estudo mostraram ainda a mesma composição em dois dos esmaltes positivos, que continham tolueno, formaldeido, dibutilftalato, resina de formaldeído e cânfora. Um deles, porém, não continha tolueno.

Com relação ao volume de produto, os frascos de esmalte considerados cheios, com média e pouca quantidade compreenderam $40,60 \%, \quad 32,67 \%$ e $26,73 \%$ das amostras 
analisadas, respectivamente. Os três esmaltes nos quais foram observados agentes fúngicos apresentaram um volume médio de produto.

Em todos os casos averiguados, os fungos foram identificados como sendo do gênero Candida spp. (Figura 1). Com a confirmação de crescimento de Candida spp. nos esmaltes, o cultivo em meio cromogênio Hicrome TM Candida diferential Agar permitiu

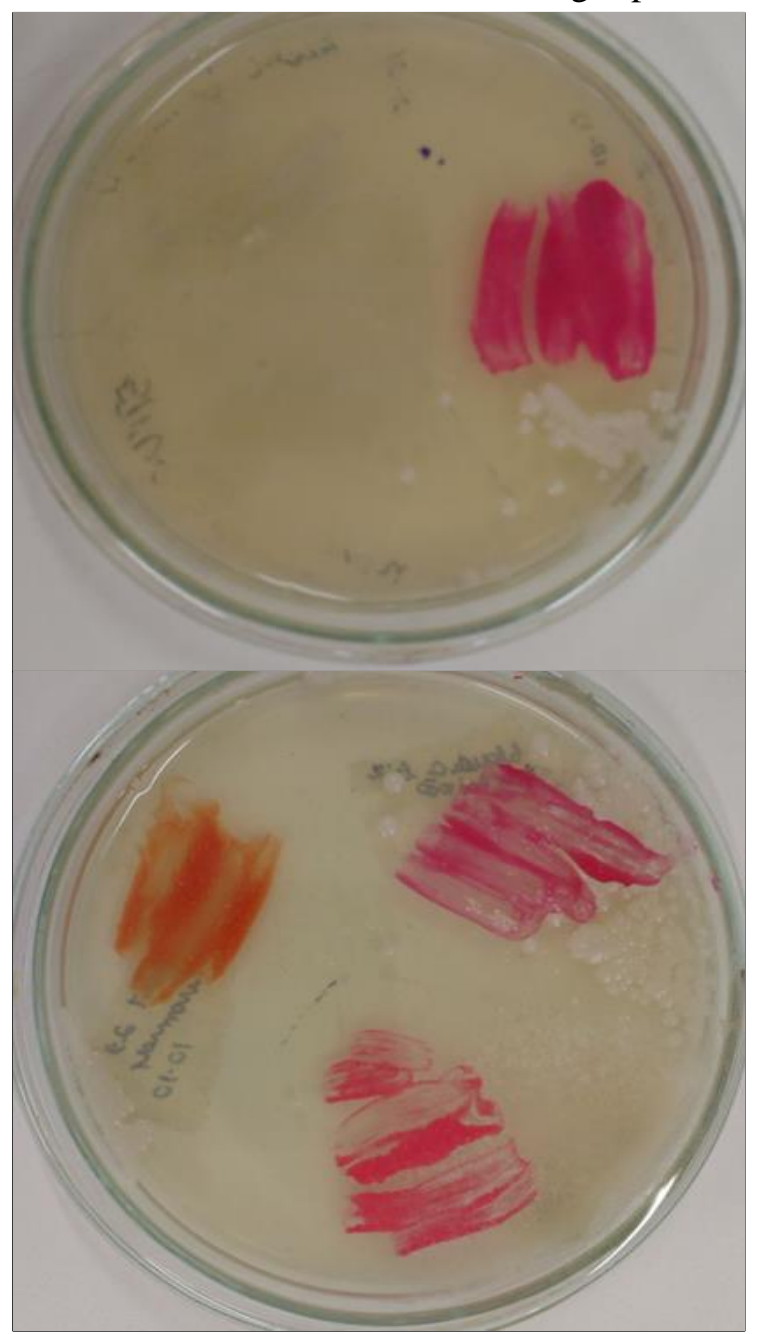

Figura 1. Crescimento de Candida spp. em meio saboraund dextrosado

a identificação da espécie Candida krusei em duas das amostras (Figura 2). Entretanto, uma das amostras não pode ser identificada, pois apresentou uma coloração duvidosa.

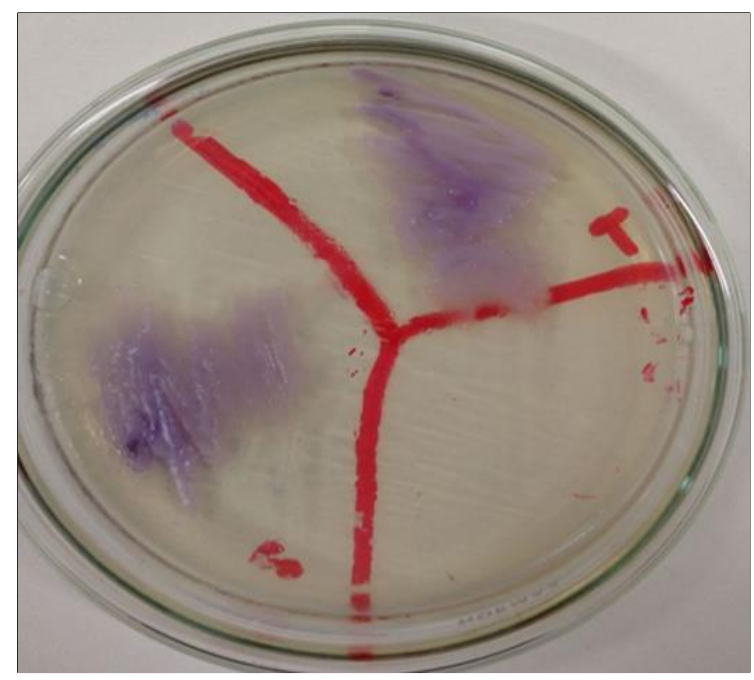

Figura 2. Diferenciação das espécies em meio cromogênico

\section{Discussão}

Atualmente, a problemática da disseminação de patógenos não está circunscrita apenas aos hospitais, se estendendo à diversos serviços $^{8}$. Nesse âmbito, as consequências da transmissão de agentes etiológicos de onicomicoses em salões de beleza variam do desconforto à perda da sensibilidade dos dedos. Outrossim, as micoses de unha podem ensejar problemas de cunho psicológico, bem como afetar a capacidade de trabalho de indivíduos acometidos 9 .

Neste inquérito, evidenciou-se o crescimento do gênero Candida spp. em 2,97\% das amostras avaliadas. Esses dados corroboram 
uma análise realizada por Almeida ${ }^{10}$, onde constatou-se a sobrevivência de quatro espécies de fungos em esmaltes, a saber: Trichophyton rubrum, Trichopyton mentagrophytes, Candida albicans e Candida parapsilosis.

No presente estudo, foi possível identificar que as leveduras de Candida isoladas em dois dos esmaltes analisados pertenciam à espécie Candida krusei, considerada uma das espécies oportunistas mais importantes. Além disso, apresenta resistência intrínseca ao fluconazol, o qual é amplamente utilizado no tratamento de infecções fúngicas ${ }^{11}$.

Observa-se uma crescente preocupação com as micoses de unha decorrentes da infecção por cepas de Candida frente ao risco de acometimento da placa ungueal ${ }^{12}$. As espécies de Candida comumente encontradas nas unhas são Candida albicans, Candida Krusei, Candida tropicalis e Candida parapsilosis ${ }^{13,14}$. Fatores como imunidade do hospedeiro, idade avançada, infecção pelo HIV, substâncias químicas e hiperhidratação contribuem para o desenvolvimento e severidade das onicomicoses por Candida ${ }^{12}$.

O tratamento das micoses de unha representa um desafio, haja vista os fatores que influenciam a instalação da doença e resposta à terapia, além dos os mecanismos de resistência aos antifúngicos. Além disso, o sucesso da terapia depende essencialmente de um diagnóstico correto ${ }^{4,12,15}$.

Ao analisar o volume contido em cada frasco, constatou-se que os três esmaltes que apresentaram resultados positivos possuíam uma quantidade média do produto. Cabe sublinhar que os esmaltes mais utilizados são, potencialmente, aqueles compartilhados por várias pessoas, o que facilita a contaminação por microrganismos. Entretanto, é necessário ressaltar que um dos esmaltes encontrava-se fora do prazo de validade. Por isso, não é possível garantir a qualidade do produto, uma vez que este fato pode ter influência sobre sua composição química, de modo que a contaminação pelo agente fúngico possa ter sido facilitada.

Durante a remoção do eponiquio, lesões provocadas por materiais não descontaminados de forma adequada podem culminar na transmissão de diversos agentes infeciosos ${ }^{16}$. Desse modo, quando os profissionais desse ramo não são devidamente preparados e, por isso, não respeitam as normas de segurança, colaboram para o aumento do risco de transmissão desses patógenos ${ }^{7,8}$. 
É imprescindível, portanto, que os objetos utilizados nesses espaços sejam esterilizados após o uso. Não obstante, para além do material perfurocortante, os resultados desta pesquisa mostraram que os esmaltes podem ser um fômite para a transmissão de fungos. Logo, os cuidados não devem se restringir aos instrumentos utilizados nos salões de beleza, mas se estender à estes produtos aplicados sobre as unhas.

\section{Conclusão}

Em suma, o crescimento do gênero Candida em esmaltes utilizados em salões de beleza demonstrou a relevância de alertar os profissionais e a população no que diz respeito à possibilidade da transmissão de fungos por meio do seu compartilhamento.

\section{Referências}

1. Criado PR, Dantas KC, Benini LV, Oliveira CB, Takiguti FA, Vasconcellos C. Micoses superficiais e os elementos da resposta imune. An Bras Dermatol. 2011;86(4):726-731.

2. Somenzi CC, Ribeiro TS, Menezes A. Características particulares da micologia clínica e o diagnóstico laboratorial de micoses superficiais. NewsLab. 2006;77:106-118.

3. Martins EA, Guerrer LV, Cunha KC, Soares MMCN, Almeida MTG. Onicomicose: estudo clínico, epidemiológico e micológico no município de São José do Rio Preto. Rev Soc Bras Med Trop. 2007;40(5):596- 598.

4. Peres NTA, Maranhão FCA, Rossi A, Martinez-Rossi NM. Dermatófitos: interação patógeno-hospedeiro e resistência a antifúngicos. An Bras Dermatol. 2010;85(5):657-667.

5. Vidigal PG, Svidzinski TI. Leveduras nos tratos urinário e respiratório: infecção fúngica ou não? J Bras Patol Med Lab. 2009;45(1):55-64.

6. Nakamura HM, Cladeira SM, AVILA MAG. Incidência de infecções fúngicas em pacientes cirúrgicos: uma abordagem retrospectiva. Revista SOBECC. 2013;18(3):49-58.

7. Wazir MS, Mehmood S, Ahmed A, Jadoon HR. Awareness among barbers about health hazards associated with their profession. J Ayub Med Coll Abbottabad. 2008;20(2):35-8.

8. Garbaccio JL, Oliveira AC. Hidden in the risk segment of aesthetic and beauty: An assessment of the knowledge of professional and practices in salons biosafety. Texto Contexto Enferm. 2013;22(4):989-998.

9. Araújo AJG, Bastos OMP, Souza MAJ, Oliveira JC. Ocorrência de onicomicose em pacientes atendidos em consultórios dermatológicos da cidade do Rio de Janeiro, Brasil. An Bras Dermatol. 2003;78(3):299-308.

10. Almeida MTG. Nail Polishes: Uncommon fomites for the transmission of pathogenic fungi. J Microb Biochem Technol. 2013;5:56.

11. Kanafani ZA, Perfect JR. Antimicrobial resistance: resistance to antifungal agents: mechanisms and clinical impact. Clin Infect Dis. 2008;46(1):120-128.

12. Jayatilake JA, Tilakaratne WM, Panagoda GJ. Candidal onychomycosis: a mini-review. Mycopathologia. 2009;168(4):165-173.

13. Crocco EI, Mimica LM J, Muramatu LH, et al. Identificação de espécies de Candida e susceptibilidade antifúngica in vitro: estudo de 100 pacientes com candidíases superficiais. An Bras Dermatol. 2004;79:689-697. 
14. Miranda KC, Araújo CRA, Khrais CHA, et al. Identificação de leveduras do gênero Candida nas unhas e em descamação de pele em Goiânia (GO), durante o ano de 2003. Ver Patol Trop 2005;34(2):123-128.

15. Ligia RBR, Chiacchio ND. Manual de conduta nas onicomicoses diagnóstico e tratamento. In: Sociedade Brasileira de
Dermatologia. Manual de Conduta. Rio de Janeiro: Sociedade Brasileira de Dermatologia. 2004;191-201p.

16. Oliveira ACDS, Focaccia R. Survey of hepatitis $\mathrm{B}$ and $\mathrm{C}$ infection control: procedures at manicure and pedicure facilities in São Paulo, Brazil. Braz J Infect Dis. 2010;14(5):502-507.

\section{Reservado aos Editores}

Data de submissão:15/02//2021

Data de aprovação: 19/03/2021 